\title{
Is Training Load Associated with Symptoms of Overuse Injury in Dancers?
}

\section{A Prospective Observational Study}

J.R.E. Boeding, $\mathrm{MD}^{1}$

E. Visser, M.A. ${ }^{2.3}$

D.E. Meuffels , MD, $\mathrm{PhD}^{1,3}$

R.J. de Vos, MD, $\mathrm{PhD}^{1}$

${ }^{1}$ Department of Orthopaedic Surgery, Erasmus Medical Centre, Rotterdam, The Netherlands ${ }^{1}$

${ }^{2}$ Sports Medical Centre - Nieuw Rotterdams Peil, Rotterdam, The Netherlands ${ }^{2}$

${ }^{3}$ Scapino Ballet, Rotterdam, The Netherlands ${ }^{3}$

Disclosure: The authors have no financial interests to declare in relation to the content of this article.

Corresponding author:

J.R.E. Boeding (jeskeboeding@gmail.com)

Erasmus MC, University Medical Centre

Department of Orthopaedic Surgery

's Gravendijkwal 230, 3015CE Rotterdam

Postbus 2040, The Netherlands

Tel.: +31 107033640 


\begin{abstract}
Overuse injuries in dance are extremely common and difficult to treat. High training load and dancing with pain are frequently regarded as risk factors for musculoskeletal injuries in professional dancers. The aims of this study were to assess for: 1) the association between training load and symptoms of overuse injury in professional dancers, and 2) any difference between the number of "time-off" injuries and injuries causing significant symptoms not leading to decreased performance time. Twenty-one dancers from a professional contemporary dance company were followed for seven weeks. They completed the dancespecific "Self-Estimated Functional Inability due to Pain" (SEFIP) questionnaire on a weekly basis to quantify musculoskeletal pain. Their training load was calculated by multiplying the Ratings of Perceived Exertion scale (RPE Borg CR10) with the daily training time. Associations between STL and SEFIP scores, recorded on a weekly basis, were evaluated using a mixed linear model with repeated measurements. No significant association was found between training load and severity of musculoskeletal pain. However, the STL of the dancers with no symptoms of overuse-injury, SEFIP $=0$, was significantly lower compared to the dancers with symptoms, SEFIP $>0 ; \mathrm{P}=0.02$. No time off because of injury was reported during the study period. There were 251 symptoms of overuse injury reported and $67 \%$ of the recorded time was danced with pain. It is concluded that dancers without musculoskeletal pain had lower training loads. While no "time-off" injuries were found, two-third of the professional dancers danced with pain during this seven-week period.
\end{abstract}




\section{Introduction}

Injuries are a fact of life in professional dance. It is widely acknowledged that overuse accounts for the majority of all dance injuries. These injuries are difficult to treat, often result in time-loss from dance activities, and can in turn lead to diminished acquisition of dance skills. In addition, overuse injuries often cause absenteeism, which can have a negative influence on dance companies from a socio-economic perspective. ${ }^{1}$

Previous studies have postulated different risk factors for dance injuries, but training load (expressed as the duration of training multiplied by the rate of perceived exertion of that training) has not been evaluated as risk factors for overuse injury. A recent study in Australian football players demonstrated that larger than normal weekly training loads and sudden training load increases are a risk factor for musculoskeletal injury. ${ }^{2}$ There is, however, a lack of evidence that training load is associated with the development of musculoskeletal injuries or symptoms of overuse injury in professional dancers.

For the purposes of this study "dance injury" refers to an anatomic tissue impairment diagnosed by a licensed health practitioner that resulted in full time loss from activity for one or more days. ${ }^{3}$ This "time-loss" definition captures only the end-stage of injuries, or the socalled "tip of the iceberg." " Due to the high dedication of dancers for their profession and art form, it may be that only a small proportion of injuries are reported in this population. Therefore, it may be important to quantify the amount of pain dancers tend to experience before "time-loss" injuries are acknowledged. It is unknown whether there is a large difference between the number of "time-off" injuries and those that cause significant symptoms but do not lead to decreased performance time. 
Therefore, this study aimed to assess the association between training load and symptoms of overuse injury in professional dancers, and the difference between "time-off” injuries and injuries causing significant symptoms not leading to decreased performance time.

\section{Materials and Methods}

\section{Study Design}

A prospective observational design was used for this study, which involved a group of 21 dancers from a professional contemporary dance company. -Before starting the study all dancers signed an informed consent form, and approval of the local medical ethics committee was obtained (MEC-2015-316).

\section{Outcome measures}

Self-estimated functional inability because of pain (SEFIP) is a dance-specific tool that is designed to register symptoms of overuse injury. ${ }^{5}$ It is particularly suitable for repeated measurements, to observe, for example, the fluctuation of symptoms throughout a season. It identifies pain and is useful as a screening instrument in dancers. ${ }^{6}$ The self-administered questionnaire scores the amount of pain in 14 body parts on a scale from "no pain" ( 0 points) to "cannot work because of pain" (4 points). The SEFIP has been well validated and is intended to be used primarily with professional dancers. To obtain a good impression of the workload involved a response rate of $80 \%$ should be achieved. ${ }^{5}$

Rating of Perceived Exertion (RPE) Borg CR10 scale is a general intensity anchor-based measure of the subjectively evaluated degree of exertion and pain in a given task. This scale, ranging from zero to ten, was used to measure the training and performance intensity of the 
dancers' daily schedule during this study. This method had been used previously in a study with Australian football (AFL) players. $^{2}$

The training load (STL) was determined by multiplying session intensity (RPE) by its duration (minutes), expressed in arbitrary units (AU), for training, performance, and other activities combined over a given day. This method was originally proposed by Foster et al. ${ }^{7}$ as a valid means to quantify sport participation in a wide variety of exercise types.

Dance Exposure (DE) is dance-related training activity; participation in class, rehearsal, or performance during which the dancers were exposed to the possibility of a dance injury.

\section{Procedures}

The researchers gave detailed instructions to all dancers before starting the study. The dancers received a form on which the RPE scale and the days of the week were printed. They were asked to score the intensity of their training and/or performance at the end of each day with the use of the RPE. In addition, they were asked to record their exercise activities beside their training schedules on the RPE form. The dancers were informed about the use of the SEFIP and were asked to complete a standardized set of baseline demographics (age, weight, gender, actual injuries, time of dancing professionally, time dancing with the company from which they were recruited for this study, and the age at which they started dancing). The principal investigator emphasized that all questionnaires had to be completed, regardless of the absence of symptoms during that day (RPE) or week (SEFIP).

Dancers received and completed the SEFIP questionnaire and the Borg-CR10 scale on a weekly basis. In cases of uncertainty, questions were discussed with the principal 
investigator. The collected data had no influence on the treatment or training load of the dancers during the study period, as the company's physiotherapists and supervisors did not have access to them.

Training schedules of each independent dancer and injuries that occurred during the study period were reported by the dancers to the principal investigator. If dancers were unable to complete scheduled training sessions they were asked to mention this or record it on their RPE questionnaire, so it could be adjusted before calculating their AU.

\section{Statistical analyses}

The association between STL and SEFIP scores was statistically evaluated with a mixed linear model. Adjustments were made for variables that influenced the outcome with $\mathrm{p}<$ 0.10. Beta coefficients (ß) and confidence intervals (CIs) were displayed for important correlations to estimate association. In cases of missing data, imputation was performed. Data were assumed to be missed at random after evaluating the pattern of missing data using the Little's MCAR test. Missing data were imputed 10 times using a multiple imputation process with SPSS software (version 23). The imputation model included all variables that had significant influence $(\mathrm{p}<0.10)$ on the outcome.

To estimate the total STL in dancers with (SEFIP > 0) and without symptoms of overuse injury $(\mathrm{SEFIP}=0)$, an independent t-test was used. The potential difference in prevalence of “time-off” injuries was evaluated as a fraction of the prevalence of injuries causing significant symptoms not leading to decreased performance time.

\section{Results}




\section{Participants}

A total of 21 professional dancers of the 22 potentially eligible dancers from the company in question agreed to participate in the study. Participants were followed for seven weeks, from approval by the ethics committee until end of the performing season. There were no dancers lost to follow-up during the course of the study. However, not all dancers completed all questionnaires, with an average weekly response rate of $86 \%$ (see Figure 1). None of the professional dancers danced without symptoms of overuse injuries during the seven-week period, meaning that every dancer included in this study experienced symptoms at one point in time during the course of the study.

Insert Figure 1 about here

\section{Baseline demographics}

Eleven men and ten women participated in the study. The mean age for men and women was 24.9 (SD 1.5) and 27.5 (SD 2.2) years, respectively. Mean body mass index (BMI) reported at baseline was 22.3 (SD 0.45) for the men and 20.0 (SD 0.30) for the women. These and other baseline demographics are shown in Table 1.

Insert Table 1 about here

\section{Association between SEFIP and Training Load}

The Little's MCAR test did not show significant differences between the participants with missing data and participants with complete data. Consequently, data were "missing completely at random," and therefore missing data were handled with multiple imputation. None of the dancers were without symptoms of overuse injury during the seven-week period; 
every dancer had at least one SEFIP > 0. To statistically evaluate the training load (STL) as a risk factor for symptoms of overuse injury, the STL and SEFIP were added in a mixed linear model. No adjustments for covariates were made, as there were no other influencing variables. There was no significant association between SEFIP and subjective load ( $\beta=$ 0.000145, $\mathrm{P}=0.127,95 \% \mathrm{CI} ;-0.00043 ; 0.00333$ : see Figure 2).

Insert Figure 2 about here

Significant difference in STL was found between dancers with symptoms (scoring SEFIP > 0) or no symptoms (scoring SEFIP = 0: $\mathrm{P}=0.02 ; 95 \%$ CI $129-1479$ ). Weekly difference between these groups is shown in Figure 3.

Insert Figure 3 about here

\section{Symptoms and time-loss injuries}

Using the SEFIP 251, symptoms of overuse injury were reported by the dancers. Eight of these symptoms were scored with an SEFIP > 2, indicating that they should have thorough examination by a physiotherapist, doctor, or other person familiar with the dancer's work, and further action should be taken. ${ }^{5}$ However, no time-loss injuries were reported during the study, which means that all participants could perform during the whole period. The most frequently reported symptom locations were the lower back (19.9\%), hips (15.5\%), and upper back (11.2\%). Eleven other locations measured with the SEFIP were collated in the "other" group, as shown in Figure 4.

Insert Figure 4 about here 


\section{Dance exposures}

All participation in class, rehearsal, or performance was registered with the use of the dancers' schedules. Because there were no time-loss injuries, dance schedules were accurate for participation in class and rehearsal. During the study period the dancers collectively had 1849 class and rehearsal moments and 264 performance exposures, resulting in a total of 2113 “dance exposures” (see Figure 5). As noted previously, eight reported symptoms of injury should have been examined by a physiotherapist or doctor familiar with dance (SEFIP > 2). ${ }^{5}$ This resulted in $8 / 2113=3.8$ symptoms of injury per 1000 dance events that should have been thoroughly examined. If all symptoms are evaluated (SEFIP > 0), a total of 251/2113 = 119 symptoms of injury per 1000 dance events were found (Figure 5).

\section{Discussion}

This is the first study showing that dancers without musculoskeletal pain have lower subjective training loads compared with dancers which dance with musculoskeletal pain. However, no statistically significant association between quantified training load and the number of symptoms due to overuse injury was shown in this study. Furthermore, the prevalence of symptoms of overuse injury is much higher than the prevalence of "time-off" injuries. This means that it is common for dancers to dance with pain, and they often continue to rehearse and perform despite having symptoms of overuse injury.

These findings have important implications for healthcare professionals working with dancers. This is the first study to investigate training load as a risk factor for symptoms of overuse injury in dance. Rogalski et al. ${ }^{2}$ assessed training and game load as a risk factor for injuries in elite Australian footballers. They also used the Borg CR10 scale to calculate the 
training load (in arbitrary units) of the athletes. These researchers concluded that larger 1 weekly loads and substantial differences in previous to current week change in load, significantly increased injury risk compared to lower training and game load ranges. There are several reasons that could contribute to the differences in association between training load and injuries when comparing our study with the Rogalski et al. data. In their study the Borg CR10 scale was estimated by athletes approximately 30 minutes following each session; in ours it was not feasible for the dancers to rate the RPE after each session, so they were asked to give a mean daily RPE score at the end of the day instead. In addition, the Australian football players were followed for a longer period (10 months), which led to a better perspective on fluctuating game and training loads. The definition of injury also differed between these studies: Rogalski et al. classified their injuries by number of missed training sessions or matches; we evaluated symptoms of overuse injury reported by the SEFIP and also registered "time-off" injuries. This enabled us to assess the fraction of symptoms of overuse injury leading to "time-off" injuries (the so-called "tip of the iceberg") which was also performed in Norwegian Olympic athletes by Clarsen et al.. ${ }^{4}$ These authors also showed a large discrepancy between the amount of diagnosed injuries and the number of symptoms reported by the athletes, respectively 40 vs. 419 .

Ours is the first study showing this discrepancy in dancers; we demonstrated a major difference between the number of time-loss injuries and symptoms reported by the dancers themselves. Based on these statistics, one could conclude that there is a low risk of musculoskeletal problems in dance. However, 1) generally speaking this is unequivocally not the case, and 2) none of the dancers in the study danced the whole period without any symptoms of overuse injury. Five dancers $(24 \%)$ rehearsed and performed the complete study period with symptoms of overuse injury. It is remarkable that the dance community accepts a 
certain number of symptoms as part of the job. The high pressure and fear of disqualification at a young age might be a reason why professional dancers are used to training and performing with pain instead of seeking medical attention and adjusting their rehearsal and performance schedule. ${ }^{8}$

The 21 dancers reported 251 symptoms over a seven-week period, and no "time-off" injuries were reported. In the dance company we studied an in-house physiotherapist is employed, and a previous study showed that this typically results in a decrease in annual injuries. ${ }^{1}$ This might be an explanation for why there were no "time-off" injuries reported; i.e., symptoms were intercepted by the physiotherapist before full-blown injuries developed. The physiotherapist might also have had a positive influence on load management due to his experience in the field. He was, however, kept unaware of the STL and SEFIP data of the dancers.

This study has some limitations. Firstly, the response rate was not 100\% (see Figure 1). Therefore, we might have to be cautious with interpretation of the data. However, there were no significant differences between the participants with missing data and participants with complete data, so this was missing "at random". This enabled us to perform multiple imputation, which is the preferred method of handling missing data. Secondly, the dancers completed an on-going day-by-day self-reported analysis of the RPE scale and the amount of exercise activities next to their training schedules. This might have restricted the accuracy of the reported data. However, in another study a series with repeated questionnaires showed a good reliability during a three-week follow-up period. ${ }^{9}$ 
A third limitation could be that the study period was too short to detect associations between training load and symptoms of overuse injury. Following the dancers over a longer study period would also include different performance cycles, which might produce more fluctuation in the training load and symptoms. This would facilitate a calculation of the acute to chronic workload ratio which could be associated with symptoms of overuse injury. ${ }^{10} \mathrm{We}$ refrained from calculating this ratio in the present study because of the relatively short study period.

More research is needed to confirm training load as a risk factor for symptoms of overuse injury. It should be performed on a larger group of professional dancers, and preferably with web-based collection of data. This would make it easier for the dancer to rate the intensity of their training and performance, and therefore result in decreased missing data. We would also suggest that it would promote accuracy if the dancers estimated the intensity approximately 30 minutes following each training session or performance. Furthermore, a more accurate training load could be provided if every dance session (including self-prescribed supplementary activities) were registered with a separate RPE score. This approach would limit the influence of the number or timing of sessions. If future research would confirm the association between the STL and symptoms of overuse injury a prevention trial could be performed with load management based on the dancer's STL as intervention. By detecting symptoms of overuse injury at an earlier stage it might be possible to limit such injuries in dancers.

\section{Conclusion}

The dancers in this study without musculoskeletal pain had lower training loads. There was, however, no statistically significant association between quantified training load and the 
number of symptoms due to overuse injury. Additionally, there was a large discrepancy between the number of symptoms and the "time-off" injuries in these dancers. All of the dancers experienced symptoms of overuse during the study period. These results suggest that the efficacy of load management should be evaluated in future studies.

\section{References}

1. Solomon R Solomon J, Micheli LJ. et al. . The "cost of injuries in a professional ballet company: A five year study. . Med Probl Perform Artists. 1999 Dec;14(4):164-9.

2. Rogalski B, Dawson B, Heasman J, Gabbett TJ. Training and game loads and injury risk in elite Australian footballers. J Sci Med Sport. 2013 Nov;16(6):499-503.

Liederbach M, Hagins M, Gamboa JM, Welsh TM. Assessing and Reporting Dancer Capacities, Risk Factors, and Injuries: Recommendations from the IADMS Standard Measures Consensus Initiative. J Dance Med Sci. 2012 Dec;16(4):139-53.

4. Clarsen B, Myklebust G, Bahr R. Development and validation of a new method for the registration of overuse injuries in sports injury epidemiology: the Oslo Sports Trauma Research Centre (OSTRC) overuse injury questionnaire. Br J Sports Med. 2013 May;47(8):495-502.

5. Ramel EM, Moritz, U. \& Jarnlo, G-B. Validation of a pain questionnaire (SEFIP) for dancers with a specially created test battery. . Med Probl Perform Art. 1999 Dec;14(4):196-203.

6. Kuorinka I, Jonsson B, Kilbom A, Vinterberg H, Biering-Sorensen F, Andersson G, et al. Standardised Nordic questionnaires for the analysis of musculoskeletal symptoms. Appl Ergon. 1987 Sep;18(3):233-7.

7. Foster C, Florhaug JA, Franklin J, Gottschall L, Hrovatin LA, Parker S, et al. A new approach to monitoring exercise training. J Strength Cond Res. 2001 Feb;15(1):10915.

8. Byhring S, Bo K. Musculoskeletal injuries in the Norwegian National Ballet: a prospective cohort study. Scand J Med Sci Sports. 2002 Dec;12(6):365-70.

9. Nederhof E, Zwerver J, Brink M, Meeusen R, Lemmink K. Different diagnostic tools in nonfunctional overreaching. Int J Sports Med. 2008 Jul;29(7):590-7.

10. Hulin BT, Gabbett TJ, Lawson DW, Caputi P, Sampson JA. The acute:chronic workload ratio predicts injury: high chronic workload may decrease injury risk in elite rugby league players. Br J Sports Med. 2016 Feb;50(4):231-6. 


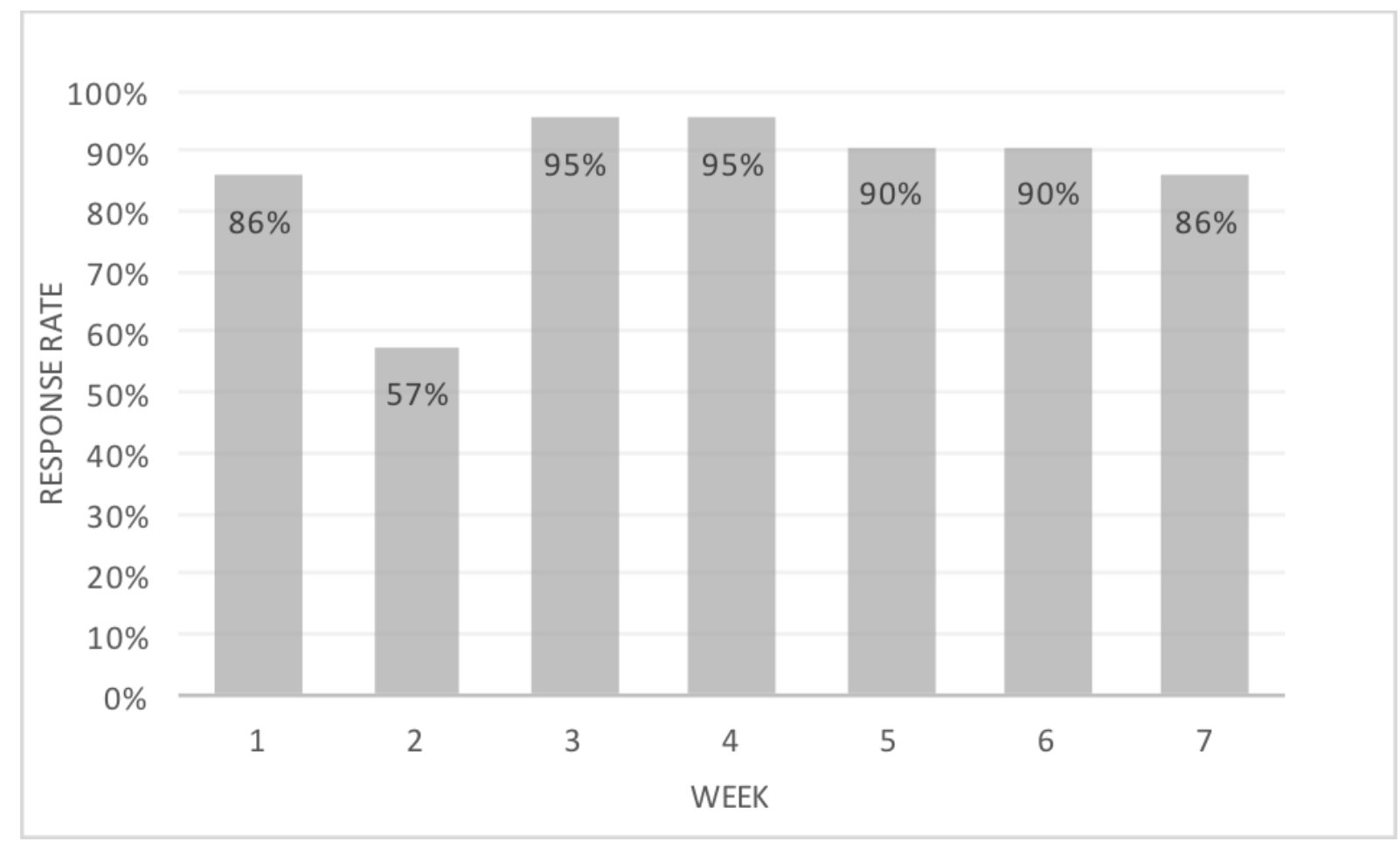

Figure 1 Response rate of the SEFIP and RPE questionnaires (weekly) 
Table 1. Baseline demographics

\begin{tabular}{lll}
\hline Baseline demographics & Men $(\mathrm{N}=11)$ & Women $(\mathrm{N}=10)$ \\
\hline Mean age, years $( \pm \mathrm{SD})$ & $24.9(1.5)$ & $27.5(2.2)$ \\
Mean height, centimeters ( $\pm \mathrm{SD})$ & $179(1.6)$ & $172(1.9)$ \\
Mean weight, kilograms ( $\pm \mathrm{SD})$ & $71.6(1.6)$ & $59(1.6)$ \\
Mean BMI $( \pm \mathrm{SD})$ & $22.3(0.5)$ & $20(0.3)$ \\
Median age started dancing, yrs. (range) & $6(6-12)$ & $5(4-7.5)$ \\
Median dancing time at dance company, mo. (range) & $18(9-35)$ & $22(9-84)$ \\
\hline SD = standard deviation; BMI = Body mass index; yrs. = years; mo. = months, \\
\hline
\end{tabular}




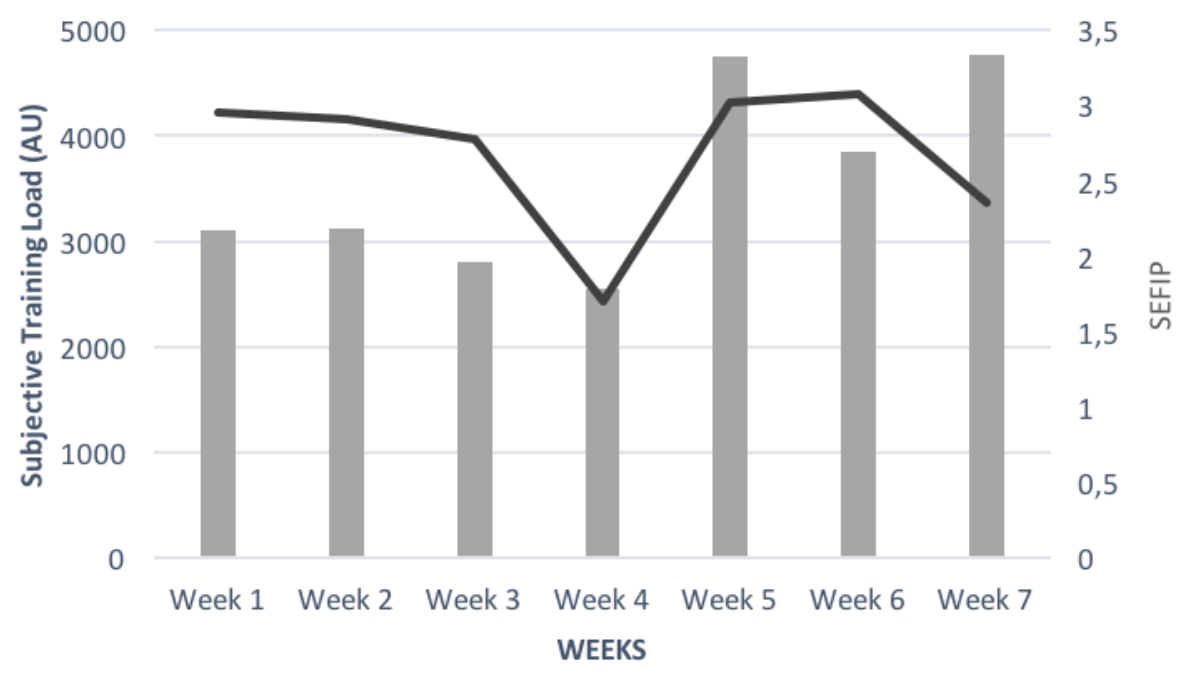

Figure 2 Mean subjective load and self-estimated functional inability of pain (weekly) Note. Black line represents SEFIP scores; grey bars represent Training Load (in Arbitrary Units) 


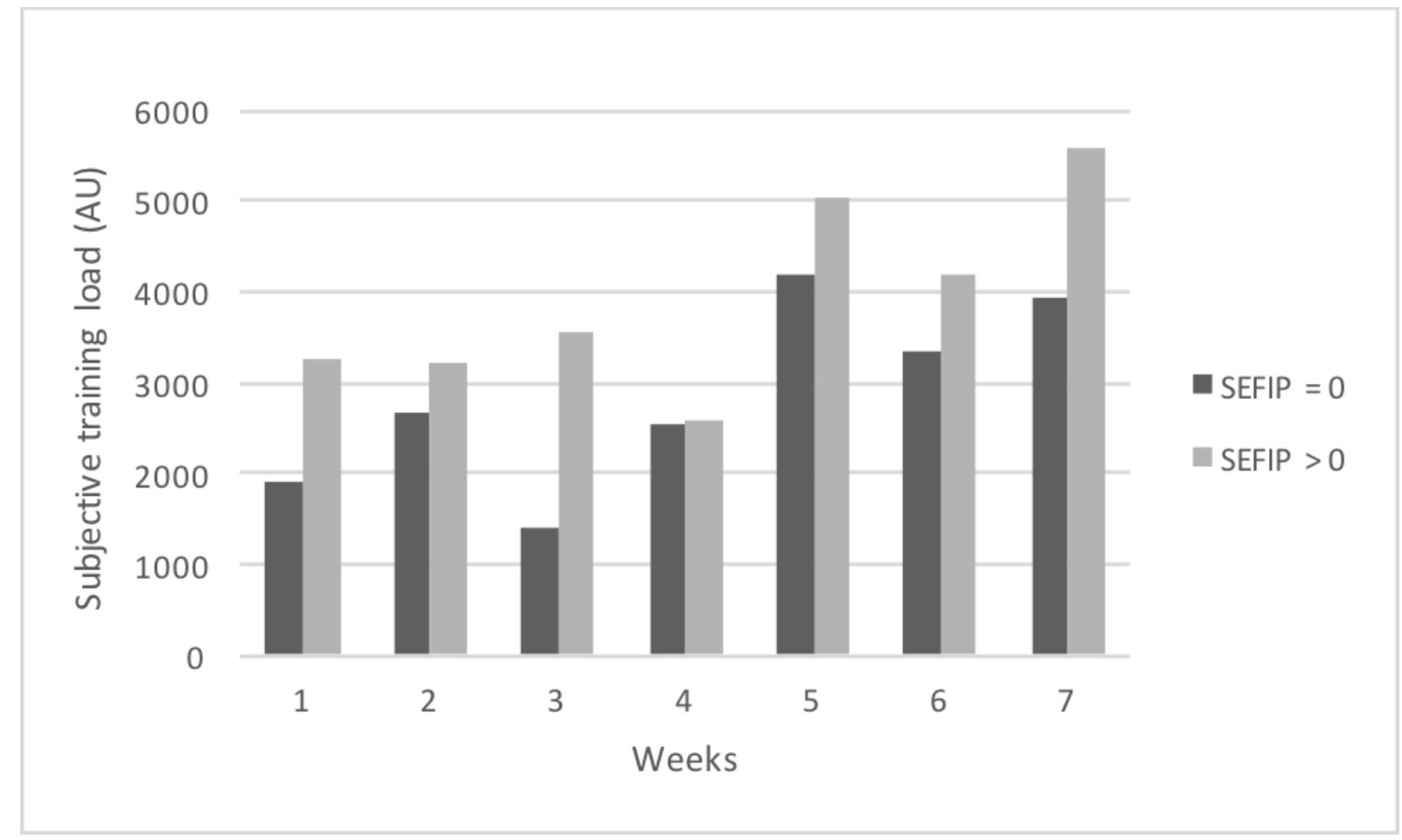

Figure 3 Difference in training load between symptomatic and asymptomatic groups (weekly) 


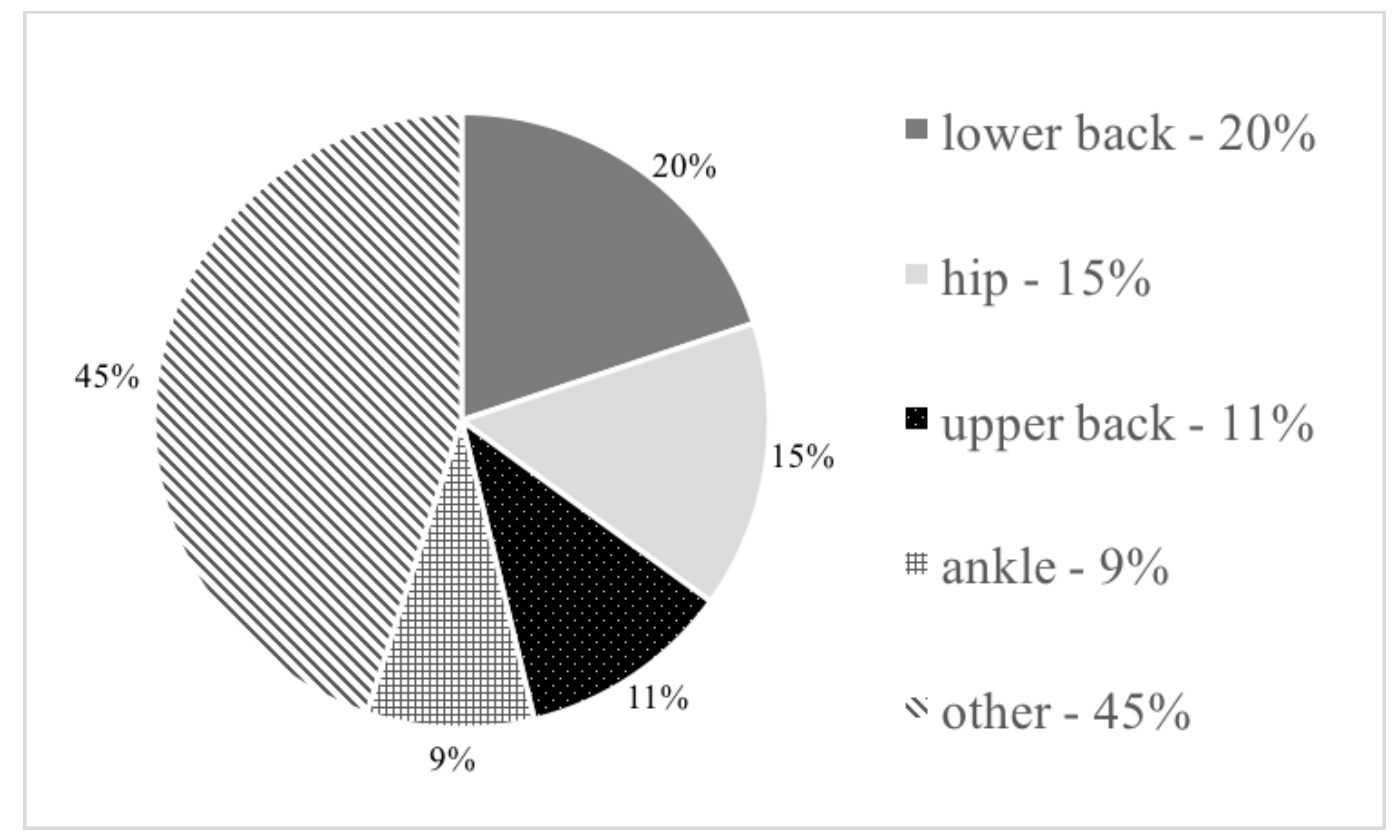

Figure 4 Location of symptoms of overuse injury 


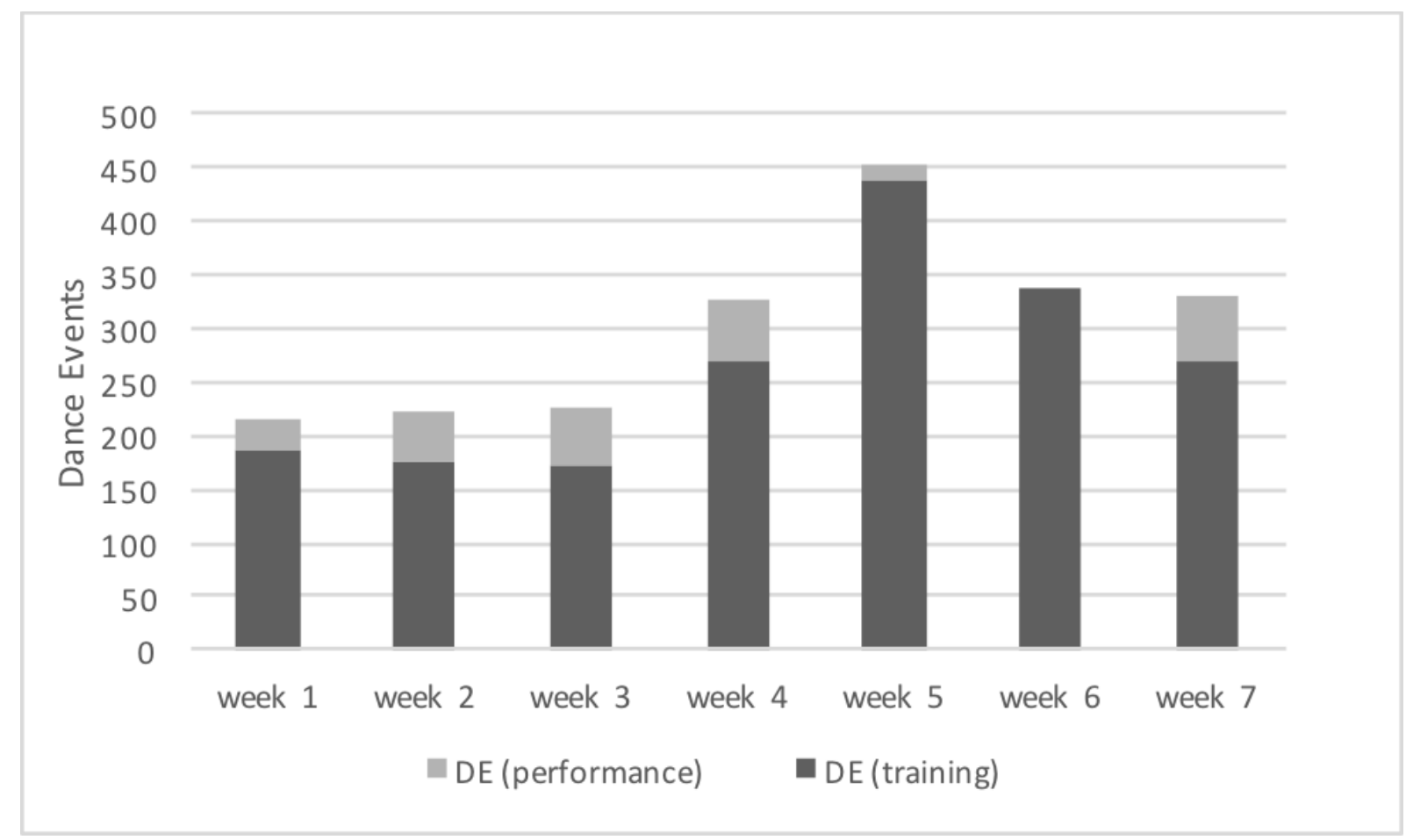

Figure 5. Dance exposure by performance and training 